\title{
Solution of the center-of-mass problem in nuclear structure calculations
}

\author{
G. Hagen, ${ }^{1}$ T. Papenbrock, ${ }^{2,1}$ and D.J. Dean ${ }^{1}$ \\ ${ }^{1}$ Physics Division, Oak Ridge National Laboratory, Oak Ridge, TN 37831, USA \\ ${ }^{2}$ Department of Physics and Astronomy, University of Tennessee, Knoxville, TN 37996, USA
}

\begin{abstract}
The coupled-cluster wave function factorizes to a very good approximation into a product of an intrinsic wave function and a Gaussian for the center-of-mass coordinate. The width of the Gaussian is in general not identical to the oscillator length of the underlying single-particle basis. The quality of the separation can be verified by a simple procedure.
\end{abstract}

PACS numbers: 21.60.-n, 21.60.Cs, 21.60.De, 31.15.bw

The atomic nucleus is a self-bound quantum manybody problem. Its Hamiltonian is invariant under translations and rotations. Thus, total momentum and total angular momentum are conserved quantities, respectively. When one chooses a single-particle basis to solve the nuclear many-body problem, one is confronted with a dilemma. There is no single-particle basis of states that are simultaneous eigenstates of the momentum operator and the angular momentum operator. For calculations of finite nuclei within the nuclear shell model, one usually chooses a spherical single-particle basis. Such a basis obviously breaks translational invariance, and the consequences have been addressed in numerous papers (see, e.g. $[1,2,[3,4,[5,[6])$.

Only two rigorous solutions to the problem are known. The first approach deals with the problem in translationally invariant Jacobi coordinates. We refer the reader to Refs 7, 8, 9] for recent applications. Due to the factorial scaling of the required antisymmetrization, this method is restricted to few-body problems with $A<7$ or so. The second approach is suitable for many-body problems but severely limits the choice of the single-particle basis to the eigenstates of the harmonic oscillator. For the $A$-body system, one considers an $A$-fermion basis that consists of all single-particle product states with excitations up to and including $N \hbar \omega$. The corresponding model space is called a full $N \hbar \omega$ space. In this space, eigenstates of the translationally invariant nuclear Hamiltonian are also eigenstates of the center-of-mass Hamiltonian $H_{\mathrm{cm}}(\omega)$ (see Eq. (3) below for a definition). Note that the Fock-space basis employed in this approach is not translationally invariant, i.e. the total momentum is not a conserved quantity. Rather, the complete $N \hbar \omega$ space ensures that all eigenstates are products of an intrinsic state $\psi_{\text {in }}$ and a center-of-mass state $\psi_{\mathrm{cm}}$. The intrinsic states are invariant under translations, while the wave function of the center-of-mass coordinate is obviously not an eigenstate of the total momentum. Thus, the factorization

$$
\psi=\psi_{\mathrm{cm}} \psi_{\mathrm{in}}
$$

is central to the $N \hbar \omega$ space, and it is essential for a correct treatment of the center-of-mass problem. Note that the particular form of the center-of-mass wave function $\psi_{\mathrm{cm}}$ is irrelevant [3, 6]. Recent examples for this approach are, e.g., the no-core shell-model calculations [10, 11] and the $0 \hbar \omega$ shell-model calculations [12, 13].

Unfortunately, the two rigorous approaches to the center-of-mass problem scale exponentially with the number of active nucleons. Furthermore, many nuclei of interest are very weakly bound, and the oscillator basis does not provide the correct radial asymptotics. Alternative wave-function-based approaches such as the coupledcluster method [14, 15, 16, 17, 18, 19, 20, 21], the unitarymodel operator approach [22], or stochastic shell-model approaches [23, 24] scale more gently with increasing size of the model space and the mass number. While these methods are also based on a single-particle basis, they do not employ a complete $N \hbar \omega$ space. Thus, there is no analytical guarantee that the wave functions computed by these methods also exhibit the factorization (10). For this reason, the results obtained by such methods are occasionally viewed with skepticism. The concern seems not primarily with the possible breaking of translational invariance, but rather with the problem to quantify the errors that might be involved. Recall that nuclear lattice calculations also break translational invariance, albeit in a controlled way [25].

It is the purpose of this Letter to eliminate these concerns. Moreover, we present a simple tool (i.e., the computation of expectation values of the generalized centerof-mass Hamiltonian) that assesses to what degree the factorization (10) is exhibited in coupled-cluster calculations. In what follows, we consider the ${ }^{16} \mathrm{O}$ nucleus and demonstrate that the coupled-cluster wave function is a product of an intrinsic wave function and a Gaussian for the center-of-mass coordinate.

For the nucleus ${ }^{16} \mathrm{O}$ we employ the low-momentum interaction $V_{\text {low }}$ [26], generated from Entem and Machleidt's chiral nucleon-nucleon potential [27] by imposing a smooth [28] momentum cutoff $\lambda=1.8 \mathrm{fm}^{-1}$. Our singleparticle basis consists of spherical oscillator states. The oscillator spacing $\hbar \omega$ and the size of the model space (in terms of the number $N$ of major oscillator shells) are the parameters of our calculations. We employ the intrinsic 
Hamiltonian

$$
\begin{aligned}
H_{\mathrm{in}} & =T-T_{\mathrm{cm}}+V, \\
& =\sum_{1 \leq i<j \leq A}\left(\frac{\left(\vec{p}_{i}-\vec{p}_{j}\right)^{2}}{2 m A}+V\left(\vec{r}_{i}-\vec{r}_{j}\right)\right) .
\end{aligned}
$$

Here, $T$ and $V$ denote the kinetic and potential energy operators, respectively, and $T_{\mathrm{cm}}$ denotes the kinetic energy of the center of mass. The intrinsic Hamiltonian is manifestly invariant under translations. We perform a spherical Hartree-Fock calculation to obtain an optimized single-particle basis. This is followed by a spherical coupled-cluster calculation [29]. Clearly, the Hartree-Fock single-particle basis in combination with the coupled-cluster method in its singles and doubles approximation (CCSD) does not employ a full $N \hbar \omega$ space. Thus, the separation (11) is not guaranteed from the outset of our calculations.

Figure 1shows the ground-state energy as a function of the oscillator spacing for a model space consisting of $N=$ 9 oscillator shells. The energy varies by about $1 \mathrm{MeV}$ while the oscillator spacing $\hbar \omega$ varies by more than a factor of two. This shows that the ground-state energy is very well converged with respect to the size of the model space. Note that we could easily employ larger model spaces and improve the convergence of our results. However, this is not necessary for the purpose of this Letter, and we refer the reader to established benchmark calculations [30].

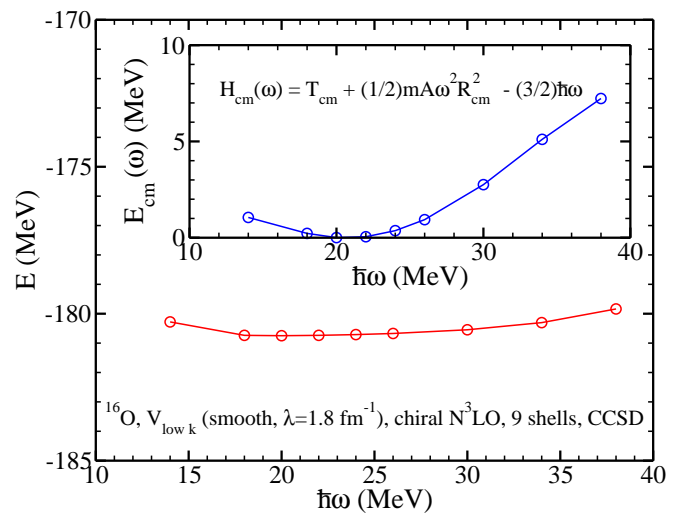

FIG. 1: (Color online) Ground-state energy (within CCSD) of ${ }^{16} \mathrm{O}$ with a low-momentum potential as a function of the oscillator spacing $\hbar \omega$. The model space consists of nine major oscillator shells. Inset: Expectation value $E_{\mathrm{cm}}(\omega)$ of the center-of mass Hamiltonian with the standard frequency dependence.

Let us consider the generalized center-of-mass Hamiltonian

$$
H_{\mathrm{cm}}(\tilde{\omega})=T_{\mathrm{cm}}+\frac{1}{2} m A \tilde{\omega}^{2} R_{\mathrm{cm}}^{2}-\frac{3}{2} \hbar \tilde{\omega} .
$$

Here, $\tilde{\omega}$ is a free parameter and not necessarily identical to the frequency $\omega$ of the underlying oscillator basis.
The generalized center-of-mass Hamiltonian (3) exhibits a zero-energy Gaussian ground-state wave function for all values of $\tilde{\omega}$. In what follows, we demonstrate that the coupled-cluster ground state is the zero-energy eigenstate of $H_{\mathrm{cm}}(\tilde{\omega})$ for a suitably chosen frequency $\tilde{\omega}$. Thus, the coupled-cluster wave function factorizes, and the centerof-mass wave function is a Gaussian.

We denote the expectation value of the generalized center-of-mass Hamiltonian (3) in the coupled-cluster ground state as

$$
E_{\mathrm{cm}}(\tilde{\omega}) \equiv\left\langle H_{\mathrm{cm}}(\tilde{\omega})\right\rangle .
$$

We compute the expectation value (4) via the HellmannFeynman theorem, i.e. we add a small perturbation $\beta H_{\mathrm{cm}}(\omega)$ with $\beta=0.001$ to the intrinsic Hamiltonian (2), and the expectation value results from the difference quotient [31, 32].

First, we consider the standard center-of-mass Hamiltonian and set $\tilde{\omega}=\omega$. The results are shown in the inset of Fig. 1. Comparing the results for the center-ofmass energy $E_{\mathrm{cm}}(\omega)$ with the ground-state energy suggests that the latter does not depend on the former. This is a first hint that the intrinsic and center-of-mass coordinates decouple. For the model space with $\hbar \omega \approx 20 \mathrm{MeV}$ the expectation value $E_{\mathrm{cm}}(\omega)$ vanishes approximately, thus indicating that the wave function factorizes. As a check, we fix $\hbar \omega=20 \mathrm{MeV}$, add the term $\beta H_{\mathrm{cm}}(\omega)$ to the intrinsic Hamiltonian, and compute the groundstate energy of the resulting Hamiltonian. We find that the ground-state energy varies by merely $15 \mathrm{keV}$ as $\beta$ is increased from zero to one. Since the intrinsic energy shown in Fig. 1 is practically independent of the frequency of the underlying oscillator basis, the wave function must approximately factorize for all values of $\hbar \omega$ shown in Fig. 1. However, only for $\hbar \omega \approx 20 \mathrm{MeV}$, do we know that the center-of-mass wave function is a Gaussian.

Let us assume that the center-of-mass wave function generally has a Gaussian shape, i.e. it is the ground state of the generalized center-of-mass Hamiltonian (3) for a suitably chosen frequency $\tilde{\omega}$. It is thus our task to determine this frequency. To this purpose, we employ the identity

$$
H_{\mathrm{cm}}(\omega)+\frac{3}{2} \hbar \omega-T_{\mathrm{cm}}=\frac{\omega^{2}}{\tilde{\omega}^{2}}\left(H_{\mathrm{cm}}(\tilde{\omega})+\frac{3}{2} \hbar \tilde{\omega}-T_{\mathrm{cm}}\right),
$$

take its expectation value, require $E_{\mathrm{cm}}(\tilde{\omega})=0$, employ $\left\langle T_{\mathrm{cm}}\right\rangle=\frac{3}{4} \hbar \tilde{\omega}$, and determine the unknown frequency $\tilde{\omega}$ from the already computed expectation values $E_{\mathrm{cm}}(\omega)$. This yields the two possible frequencies

$$
\hbar \tilde{\omega}=\hbar \omega+\frac{2}{3} E_{\mathrm{cm}}(\omega) \pm \sqrt{\frac{4}{9}\left(E_{\mathrm{cm}}(\omega)\right)^{2}+\frac{4}{3} \hbar \omega E_{\mathrm{cm}}(\omega)} .
$$

We employ these frequencies in the generalized centerof-mass Hamiltonian and compute the corresponding expectation values $E_{\mathrm{cm}}(\tilde{\omega})$. We find that one expectation 
value is close to zero, while the other is usually very large. The small expectation values are shown in Fig. 2, and the corresponding frequencies $\tilde{\omega}$ are shown in the inset of Fig. 2, The expectation values are very small compared to the energy $\hbar \tilde{\omega} \approx 20 \mathrm{MeV}$ of spurious center-of-mass excitations. The practically vanishing expectation values demonstrate that the coupled-cluster wave function factorizes. This is the main result of this Letter. The reader should not be concerned about the fact that some of the expectation values shown in Fig. 2 assume small negative values (of size $-0.01 \mathrm{MeV}$ ). Recall that the coupled-cluster method is non-variational when the cluster operator is truncated, and that small negative expectation values are thus tolerable. As shown in the inset of Fig. 2 the frequency $\tilde{\omega}$ corresponding to the Gaussian center-of-mass wave function varies only little as the frequency $\omega$ of the underlying oscillator basis is changed. We made the following two checks. First, we computed the expectation value of $T_{\mathrm{cm}}$ and found that $T_{\mathrm{cm}} \approx \frac{3}{4} \hbar \tilde{\omega}$, as expected for a Gaussian. Second, we repeated the calculations directly in the oscillator basis and did not employ the HartreeFock basis. We again find very small values for $E_{\mathrm{cm}}(\tilde{\omega})$ of order $-0.1 \mathrm{MeV}$, and an almost constant frequency $\tilde{\omega}$ very close to what we found in the Hartree-Fock basis.

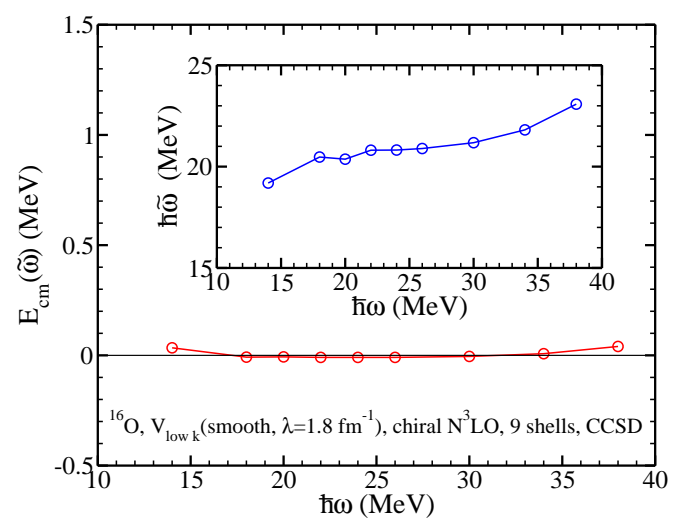

FIG. 2: (Color online) ${ }^{16} \mathrm{O}$ ground-state expectation value $E_{\mathrm{cm}}(\tilde{\omega})$ (within CCSD) of the generalized center-of-mass Hamiltonian $H_{\mathrm{cm}}(\tilde{\omega})$ as a function of the oscillator spacing $\hbar \omega$. The model space consists of nine major oscillator shells. Inset: Relation between the frequency $\tilde{\omega}$ and the frequency $\omega$ of the underlying oscillator basis.

Let us also consider interactions with higher momentum cutoff. We employ Entem and Machleidt's chiral nucleon-nucleon interaction [27] at next-to-next-to-nextto-leading order $\left(\mathrm{N}^{3} \mathrm{LO}\right)$. This interaction has an approximate high-momentum cutoff of $\lambda \approx 500 \mathrm{MeV}$. We apply the coupled-cluster method to compute the ground-state energy of ${ }^{16} \mathrm{O}$. Due to the relatively high momentum cutoff of the interaction, the wave function is more correlated. This requires us to employ a large model space and three-particle-three-hole cluster amplitudes to obtain converged solutions. The model spaces consist of
$N=19$ oscillator shells; however, the maximal singleparticle orbital angular momentum is kept at $l \leq 13$. We have verified that this is sufficient for a convergence of the results. For the three-particle-three-hole clusters, we employ the $\Lambda-\operatorname{CCSD}(\mathrm{T})$ approximation [33, 34].

The bottom part of Fig. 3 shows that the ground-state energy is practically independent of the oscillator frequency of the underlying single-particle basis for a large frequency range. This demonstrates that the results are well converged with respect to the size of the model space. We find that the expectation value $E_{\mathrm{cm}}(\omega)$ of the standard center-of-mass Hamiltonian increases with increasing frequency $\omega$ of the model space, and that it assumes values of tens of $\mathrm{MeV}$ and varies strongly with the frequency $\omega$ of the underlying oscillator basis. Clearly, the intrinsic ground-state energy is independent of the expectation value $E_{\mathrm{cm}}(\omega)$, and this suggests again a decoupling of the intrinsic and the center-of-mass wave functions.

Let us again assume a Gaussian shape for the centerof-mass wave function and follow our two-step procedure. First, we employ Eq. (5) and compute the two possible frequencies $\tilde{\omega}$ that are consistent with the already computed expectation value $E_{\mathrm{cm}}(\omega)$. Second, we compute the two corresponding expectation values $E_{\mathrm{cm}}(\tilde{\omega})$. As before, we find a large and a small expectation value for each frequency $\omega$ of the underlying oscillator basis. The small expectation values are shown in the top part of Fig. 3. The corresponding frequencies $\tilde{\omega}$ are shown in the middle part of Fig. 3. While not as impressive as for the low-momentum interaction, the expectation values $E_{\mathrm{cm}}(\tilde{\omega})$ are below $1 \mathrm{MeV}$ in size, and much smaller than the binding energy of ${ }^{16} \mathrm{O}$ or any of its excitations. In particular, the expectation values are small compared to the energy $\hbar \tilde{\omega} \approx 16 \mathrm{MeV}$ of the spurious center-of-mass excitations. A simple two-level model yields that the wave function has about $6 \%$ squared overlap with spurious states. The relative small negative eigenvalues are again due to the non-variational character of the coupledcluster method, and they are tolerable within the overall accuracy of the calculation. Thus, we can conclude that the coupled-cluster wave function also exhibits an approximate factorization (11) for the interaction with a relatively high momentum cutoff. We speculate that quadruple cluster amplitudes would be necessary to further reduce the magnitude of $E_{\mathrm{cm}}(\tilde{\omega})$. Note that the frequency $\tilde{\omega}$ that determines the width of the Gaussian center-ofmass wave function is again almost constant over a wide range of frequencies of the underlying oscillator basis.

Finally, we employed the chiral potential also for ${ }^{4} \mathrm{He}$ and show the results in Fig. 4. The bottom part shows the ground-state energy $(E \approx-25.56 \mathrm{MeV}$ in $\Lambda$ $\operatorname{CCSD}(\mathrm{T})$ ) and compares it to virtually exact FaddeevYakubowski calculations $(E \approx-25.41 \mathrm{MeV}[35])$. The middle part shows the frequency of the approximate Gaussian center-of-mass wave function, while the top part shows the expectation value $E_{\mathrm{cm}}(\tilde{\omega})$. Again, the 


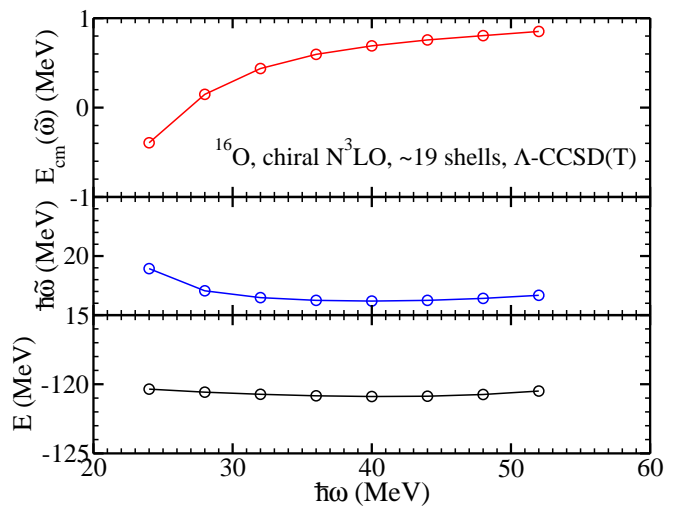

FIG. 3: (Color online) Bottom: ground-state energy of ${ }^{16} \mathrm{O}$ within the $\Lambda$-CCSD(T) approximation as a function of the frequency $\hbar \omega$ of the underlying oscillator basis. Middle: Relation between the frequency $\tilde{\omega}$ and the frequency $\omega$ of the underlying oscillator basis. Top: Expectation value $E_{\mathrm{cm}}(\tilde{\omega})$ of the center-of-mass vs. $\hbar \omega$.

approximate factorization is very statisfactory. At this moment, we have no profound understanding of the observed factorization.

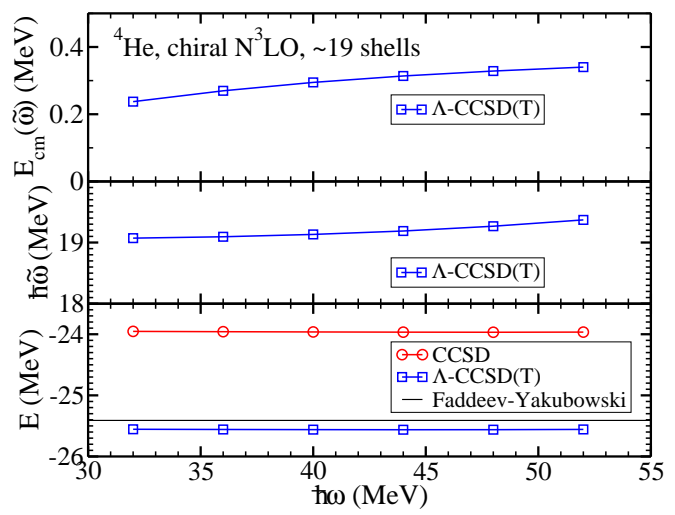

FIG. 4: (Color online) Same as Fig. 3, but for ${ }^{4} \mathrm{He}$.

In summary, we presented strong numerical evidence that wave function of the atomic nucleus is a product of an intrinsic and a center-of-mass wave function in a sufficiently large model space even when the model space is not a complete $N \hbar \omega$ oscillator space. The center-ofmass wave function is approximately a Gaussian whose width varies little with the frequency $\omega$ of the underlying oscillator basis. The reported results open the door for a verifiable description of translationally invariant states for a large variety of model spaces and many-body methods.

We acknowledge discussions with P. Navrátil, R. Roth, and S. Quaglioni that motivated this research. We thank the Institute for Nuclear Theory at the University of Washington for its hospitality during the program Effective Field Theories and the Many-Body Problem. This work was supported by the U.S. Department of Energy under Contract Nos. DE-AC05-00OR22725 with UT-Battelle, LLC (Oak Ridge National Laboratory (ORNL)), under Grant No. DE-FG02-96ER40963 (University of Tennessee (UT)), and under DE-FC0207ER41457 (UNEDF SciDAC Collaboration). This research used computational resources of the National Institute for Computational Sciences (UT/ORNL) and the National Center for Computational Sciences (ORNL).

[1] J. P. Elliott and T. H. R. Skyrme, Proc. Roy. Soc. Lon. A 232, 561 (1955).

[2] H. J. Lipkin, Phys. Rev. 110, 1395 (1958).

[3] C. M. Vincent, Phys. Rev. C 8, 929 (1973).

[4] D. H. Gloeckner and R. D. Lawson, Phys. Lett. B 53, 313 (1974).

[5] J. B. McGrory and B. H. Wildenthal, Phys. Lett. B 60, 5 (1975).

[6] R. R. Whitehead et al., Adv. Nucl. Phys. 9, 123 (1977).

[7] R. F. Bishop et al., Phys. Rev. C 42, 1341, (1991).

[8] A. Nogga, et al., Phys. Rev. C 65, 054003 (2002).

[9] S. Bacca et al., Phys. Rev. Lett. 102, 162501 (2009).

[10] P. Navrátil, J. P. Vary, and B. R. Barrett, Phys. Rev. C 62, 054311 (2000).

[11] P. Navrátil et al., arXiv:0904.0463.

[12] E. Caurier et al., Rev. Mod. Phys. 77, 427 (2005).

[13] Michio Honma et al., Phys. Rev. C 69, 034335 (2004).

[14] F. Coester, Nucl. Phys. 7, 421 (1958).

[15] F. Coester and H. Kümmel, Nucl. Phys. 17, 477 (1960).

[16] J. Čížek, J. Chem. Phys. 45, 4256 (1966).

[17] J. Čížek, Adv. Chem. Phys. 14, 35 (1969).

[18] H. Kümmel, K.H. Lührmann, and J.G. Zabolitzky, Phys. Rep. 36, 1 (1978).

[19] B. Mihaila and J.H. Heisenberg, Phys. Rev. Lett. 84, 1403 (2000).

[20] D.J. Dean and M. Hjorth-Jensen, Phys. Rev. C 69, 054320 (2004).

[21] R.J. Bartlett and M. Musiał, Rev. Mod. Phys. 79, 291 (2007).

[22] Shinichiro Fujii, Ryoji Okamoto, and Kenji Suzuki, Phys. Rev. C 69, 034328 (2004).

[23] S. E. Koonin, D.J. Dean, K. Langanke, Phys. Rep. 278, 1 (1997).

[24] T. Otsuka et al., Prog. Part. Nucl. Phys. 47, 319 (2001).

[25] Dean Lee, arXiv:0804.3501

[26] S. K. Bogner, T. T. S. Kuo, and A. Schwenk, Phys. Rep. 386, 1 (2003).

[27] D. R. Entem and R. Machleidt, Phys. Rev. C 68, 041001(R) (2003).

[28] S. K. Bogner et al., Nucl. Phys. A 784, 79 (2007).

[29] G. Hagen et al., Phys. Rev. Lett. 101, 092502 (2008).

[30] G. Hagen, et al. Phys. Rev. C 76, 044305 (2007).

[31] G. H. F. Dierksen, B. O. Roos, and A. J. Sadlej, Chem. Phys. 59, 29 (1981).

[32] M. Enzerhof, C. M. Marian, and S. D. Peyerimhoff, Chem. Phys. Lett. 204, 59 (1993).

[33] S. A. Kucharski and R. J. Bartlett, J. Chem. Phys. 108, 5243 (1998).

[34] A. D. Taube and R. J. Bartlett, J. Chem. Phys. 128, 044110 (2008). 
[35] P. Navrátil and E. Caurier, Phys. Rev. C 69, 014311 (2004). 